\title{
Orthopaedic Care of Children with Myelomeningocele: a Modern Programme of Rehabilitation
}

\author{
E. H. STRACH,* M.CH.ORTH., F.R.C.S.
}

Brit. med. 7., 1967, 3, 791-794

"A niche of usefulness and self-respect exists for every man, however handicapped, but that niche must be found for him." -JoHn GaLsworThy.

An ever-increasing number of children suffering from congenital paraplegia due to myelomeningocele are being referred to the orthopaedic surgeon for treatment of skeletal deformities and for rehabilitation. The management of these children is often so complex that it presents a veritable challenge to the orthopaedic surgeon. The aim of this paper is to show some of the problems he has to deal with and to suggest some lines of management.

This study is based on personal experience with 173 patients (98 male, 75 female) who were referred for orthopaedic treatment during the past 10 years. They represent, therefore, a selected group of cases, but they are a typical cross-section of cases seen in the orthopaedic department.

I must emphasize that the intensive programme of rehabilitation described here is not accepted by all workers in this field.

The ages of the patients at the time of review or at the time of death were: under 5 years, $104+10$ deaths ; 5-10 years, $45+1$ death ; 10-17 years, $12+1$ death. The primary myelomeningocele operation was performed in the first 24 hours in 81 ; at 2 to 7 days in 44 ; at 8 days to 6 months in 24 ; and later in 11. The timing of the operation in two patients was unknown and in 11 cases operation was not performed.

Hydrocephalus was present in 130 cases $(75 \%)$. Ventriculoatrial shunt operation was performed in the first month of life in 54 children and at a later date in 65 children. Spontaneous arrest occurred in 11 cases.

Paralysis of the legs was severe in 89 children (above the twelfth dorsal segment), moderate in 43 (above the fifth lumbar segment), slight in 15 (at or below first sacral segment), and spastic in 8. Paralysis was not present in 18 children.

Urinary diversion was performed in 23 cases.

Size of the Problem.-There is no increase in the number of children born with this malformation. With modern initial treatment of the myelomeningocele and the hydrocephalus many more children survive than formerly. The incidence of myelnmeningocele in the United Kingdom is 2.5 to 3 per 1,000 live births. It has been estimated that in England and Wales 2.500 children are born every year with this malformation. Fifty per cent. will reach the age of 5 and a quarter of these will have no handicap and three-quarters will have a physical or mental defect. Eighty-four per cent. of all survivors are mentally normal.

\section{Early Management}

This is usually carried out by the paediatric surgeon in a neonatal surgical unit. Ideally, the myelomeningocele should be closed within 24 hours of birth to prevent infection and to preserve neural tissue. The frequent occurrence of hydrocephalus can be controlled by ventriculo-atrial drainage by means of a Holter valve. There are complications, but it is important to realize that the control of hydrocephalus is successful and without complications in two-thirds of the cases.

\section{Orthopaedic Treatment}

The aim is towards achieving independence of the congenitally paralysed child, notably to enable it to stand and to walk. There are cases where this aim is unobtainable; where there is severe mental deficiency, rehabilitation will fail. It is interesting to note than only $8 \%$ of all reviewed cases showed a severe degree of mental deficiency. Another $8 \%$ showed a milder degree, and in some of these rehabilitation was successful and even improved the child's mental capacity. It seems that physical improvement gives rise to an increased mental performance. Other factors militating against a successful rehabilitation are lack of determination of parents, a large head, and obesity.

\section{Early Orthopaedic Management}

In the first few weeks of life the orthopaedic management will take second place to the treatment of the spinal defect and hydrocephalus. However, an assessment of the neurological lesion and deformities should be done on the day of birth and repeated during the first few weeks of life. Muscle charting of the neonate is not an easy matter and often only a rough estimate of the activity of muscle groups is possible. Electromyography has been used but so far has proved of only academic interest. With experience, muscle testing becomes easier. Sharrard (1964) has rightly pointed out that the normal neonate moves his limbs freely while awake, and failure to move the legs spontaneously indicates a severe degree of paralysis. It is important to differentiate between spontaneous and reflex movements. The latter occurs in the isolated cord syndrome below the level of the lesion and is quite a common occurrence.

The neurological lesion is often segmental, and paralytic deformities occur according to the level of the lesion. For example, if innervation is intact down to the fourth lumbar segment and absent below it, the hips will be flexed and adducted, the knees will be extended, and the feet dorsiflexed.

Besides paralytic deformities there may be concomitant truly congenital deformities such as hemivertebrae, rib abnormalities, severe club-feet, and congenital dislocation of the hip. In the

* Consultant Orthopaedic Surgeon, St. Helens and Whiston Hospitals (Liverpool Regional Hospital Board). 
earlier stages these deformities can be controlled by conservative means, such as repeated moulding, retentive strapping, and plaster slabs or splints. There is, of course, a danger of sores developing in these insensitive legs, but a well-padded plaster or splint can be applied with impunity.

\section{Later Treatment}

When the child reaches the age of 14 to 18 months the orthopaedic surgeon's efforts will be directed towards ambulation. It is important that ambulation should be started at an early age, for it is physiological for the walking and standing reflex patterns to develop at that time. There will be a limited

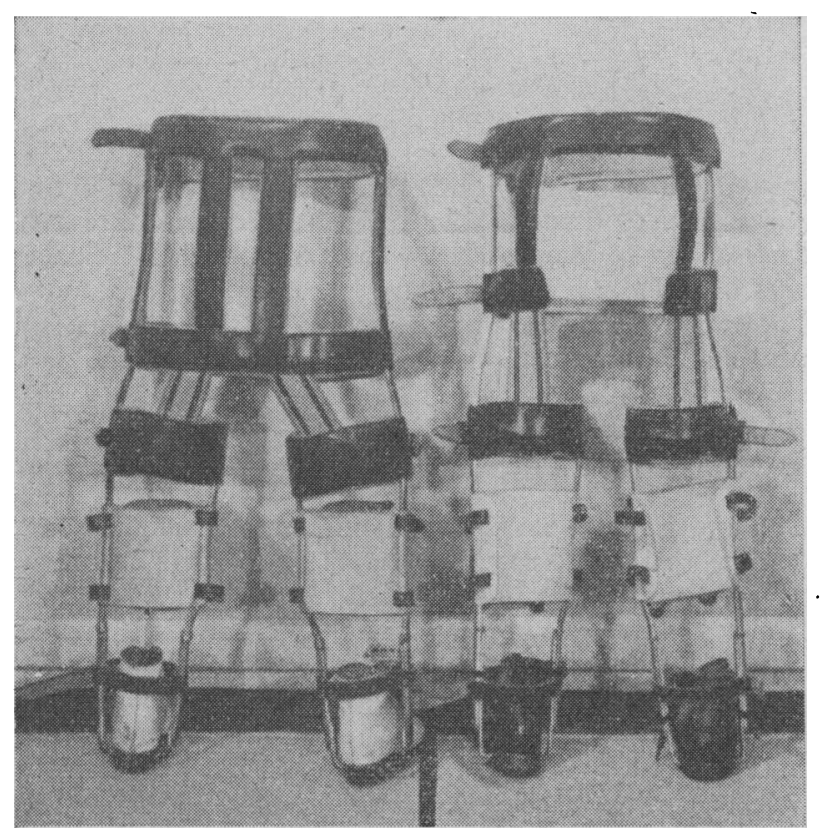

Fig. 1.- Two walking-appliances. The one on the right has a broad elastic sheet at the back to avoid pressure on any kyphosis.

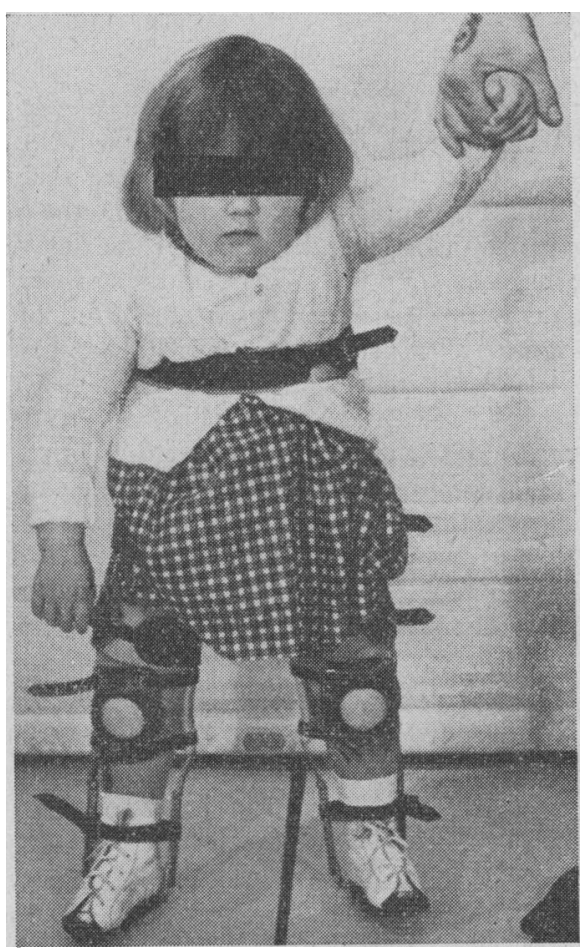

FIG. 2.-Complete paraplegia at the level of the eleventh dorsal segment in a child aged 3 . Started to walk with the appliance at age of 15 months. Normally the trunk part of the appliance is hidden by her dress. reflex pattern if lower-limb innervation is deficient or absent, but at least compensatory reflex action will be allowed to develop in the upper limbs and trunk. Theoretically, all paraplegics should be able to walk in one way or another, provided their legs are adequately supported and they can use their upper limbs. This applies to congenital paraplegia as much as to adult traumatic paraplegia. In the former there is no previous experience in walking; on the other hand, trophic sores are not as prone to develop as in adults. Early ambulation reduces the subsequent incidence of coxa valga and of trophic ulcers.

If there is complete and flaccid leg paralysis this task is simplified. There are no deformities, and all that is needed is to support the legs by an appliance which consists of two callipers joined to a body brace (Figs. 1 and 2). The children take to it readily and often protest when it is taken off. At first they are trained to stand in it for short periods. Additional support is given by holding their hands and allowing the children to grip so that they learn to use their hands purposefully for balancing and progression. They soon acquire a rocking type of gait. The appliance is worn for increasing periods during the day so that the skin can get used to the pressure. Sores are rare. The feet and any kyphosis are the areas at risk. It is useful to abduct the appliance at the level of the hips for several reasons. It gives a wider base and an increased side-to-side stability. It increases the excursion of the rocking movements during progression. If the hips tend to dislocate, the abducted position increases their stability. At times it was found useful to attach skis to the appliance, as this increases the front-to-back stability and gives the child confidence in the initial stages of walking.

As the child grows older a new appliance will have to be made, and this will contain hinges at the level of the hips to allow sitting. In some cases less rigid supports can be used as a lively splint in the form of a coil-spring appliance (Fig. 3). They allow the legs to swing at the hips. In all appliances a broad elastic strap passes over the buttocks to confer some extension to the hips. These straps may be extended to the heel in calf paralysis.

When the child is 5 knee hinges should be fitted to the appliance to allow more natural sitting, and, as the upper limbs become more efficient in balancing, the trunk part of the appliance can be replaced by a simple pelvic band.

Conventional callipers, the mainstay of the support in cases of poliomyelitis, are useless in spina bifida. They are used mainly for quadriceps paralysis, but in spina bifida isolated quadriceps paralysis is unknown. In a lesion at or above the second lumbar segment there will be quadriceps paralysis, but at the same time the glutei (which are innervated by the lower lumbar and sacral segments) will be paralysed, so that the calliper without a body brace will be insufficient. In a lesion at the fourth lumbar level the quadriceps will be working, so a calliper alone is unnecessary. The glutei will still be paralysed, and only a full appliance can replace the action of body support in these cases. Sharrard's posterior iliopsoas transfer is of immense value. It restores muscle balance around the hip and prevents dislocation, and if successful in restoring strong hip extension the child can keep the trunk upright and manage to walk without the full appliance.

\section{Hip in Spina Bifida}

(1) Flexion deformity is the most important hip deformity because it may severely hinder rehabilitation, thus making standing or walking impossible. It is caused by unequal muscle pull or by faulty posture. Most of these children attain a characteristic sitting posture with hips acutely flexed and externally rotated, with knees flexed, supporting themselves by the hands in front (Fig. 4). If they are allowed to sit like this while awake and to sleep on their sides with the hips bent, the flexion deformity will become fixed. It may yield to conserva- 
tive treatment, but if present for a long time operative flexor release may be the only hope of enabling the child to stand upright. In this series flexion deformity was present in 25 hips of 16 children. Six hips required the flexor release operation.

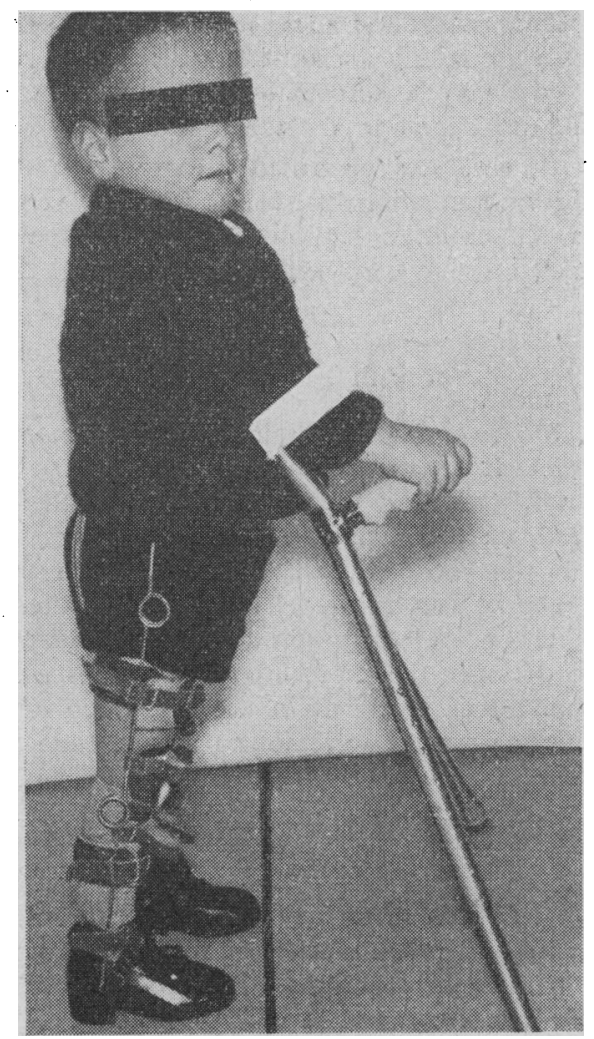

FIG. 3.-Child aged 4, with paralysis at third lumbar segment, using coil-spring appliance.

(2) External rotation deformity of the hips rarely interferes with ambulation and can be controlled by the appliance. Occasionally, one may have to transfer the sartorius to the outer side of the knee.

(3) Abduction deformity results from faulty sitting posture or from prolonged treatment of the dislocated hip, or it may be part and parcel of tightness of the tensor fasciae femoris. If postural it may respond to traction, but the tensor fasciae

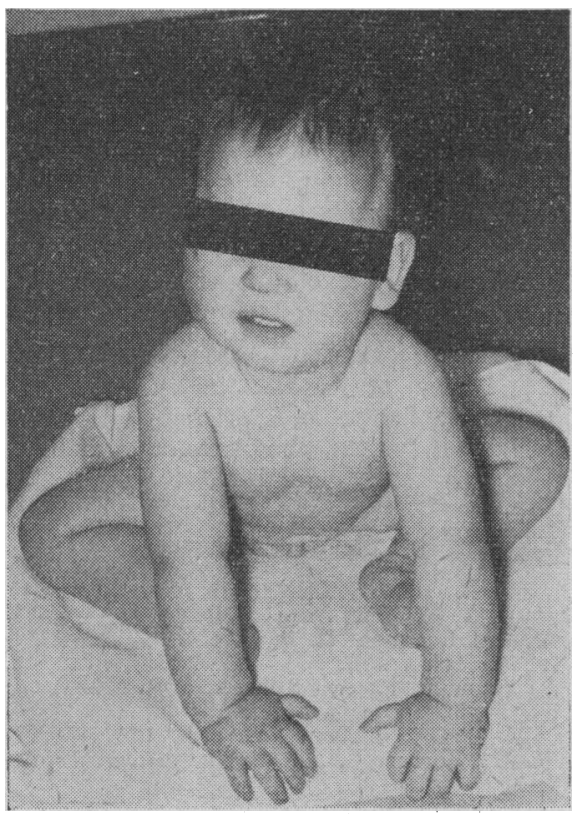

FIG. 4.-Typical sitting posture in severe congenital paraplegia (level ninth dorsal segment). femoris was a potent cause of this deformity in 13 hips, and in five cases operative release was required. If associated with coxa valga it can be corrected by varus osteotomy.

(4) Dislocation of the hip is common-103 dislocations or subluxations were found in 80 children ( $46 \%$ of all children were affected). There are four factors which can cause the dislocation:

(a) Laxity of the capsule is often present in any neonate but is much more pronounced if the hip muscles are paralysed. This factor was found in 24 cases.

(b) Unequal muscle pull is the most important single factor causing dislocation of the hip. In a lesion of the third or fourth lumbar segment the flexors and adductors are working while abductors and extensors are paralysed. The resulting adduction deformity leads to dislocation of the hip. In such cases the posterior iliopsoas transfer operation is the logical treatment of this muscle imbalance. It was present in 51 cases and in three cases of predominant spasticity.

(c) Coxa valga often occurs if the hip muscles are paralysed. It seems to be caused by the absence of the moulding force of the muscles and lack of weight-bearing. It predisposes to subluxation and later to a dislocation, which tends to occur in the slightly older child. It was present in 19 hips and in two cases with predominant spasticity.

(d) Severe dysplasia of the hip (Putti's embryonal dysplasia) was present in five cases. It was characterized by high dislocation present at birth and stiff hips, the picture resembling arthrogryposis. It was resistant to all forms of conservative treatment. In two cases open reduction of the hip was successful.

\section{Treatment of Dislocated Hips}

In the first few months a simple abduction splint, a modified Forrester-Brown splint, may be all that is required (Fig. 5). It was successful in 33 hips, though four of these were too recent to be sure about lasting success. In 28 hips dislocation recurred after such conservative treatment. In 29 cases adductor tenotomy was required. There were 16 psoas transfer operations ; nine of these also had a varus osteotomy (five of them at the time of the psoas transfer). All but one hip remained reduced, and in five cases of psoas transfer the full walking appliance was replaced by below-knee irons. Eighteen varus and rotation osteotomies were performed. Three open reductions were necessary.

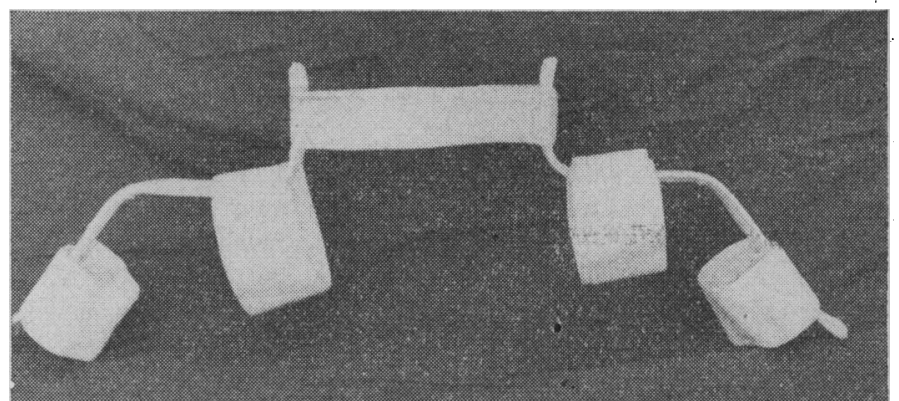

FIG. 5.-Modified Forrester-Brown splint. Vinyl-covered malleable steel bands. The connecting steel band lies in front of the abdomen, so that no pressure is exerted on the scar of the back. Velcro fasteners.

\section{Foot Deformities in Spina Bifida}

A deformity of one or both feet was present in 117 children. In addition, 25 had flail feet. The equinus element was present in 58 cases ( 91 feet), the calcaneus element in 54 cases ( $77 \mathrm{feet}$ ), the valgus element in 40 cases ( $58 \mathrm{feet}$ ), and the varus element in 35 cases ( 50 feet).

Most of the foot deformities were due to muscle imbalance, but the equinus deformity could not be explained by a segmental lesion. The equinus feet can result from spasticity or fibrosis of the calf, but in some cases it was due to an associated congenital club-foot deformity: 
The conservative treatment should be started early, due care being taken to protect the skin. In cases of muscle imbalance correction of the foot deformities is easy, but relapse occurs readily; therefore a tendon transfer operation is required to restore muscle balance. This was done in 16 feet. Tendon transplants through the interosseous membrane were found particularly successful. There were four tibialis posterior transfers to the front and outer side of the foot for the varus deformity, and also six tibialis anterior transfers to the tendo achillis insertion for the common calcaneus deformity. Elongation of the tendo achillis was performed in 30 feet. In the severe congenital club-foot deformity a radical approach was adopted because weight-bearing on the deformed foot tended to cause pressure sores. A soft-tissue release was done on six feet, and wedge tarsectomy was performed in one " intrinsically vicious club-foot" at the age of 3 .

If a child with only a mild paralysis develops an increasing varus deformity of the foot, or if the paralysis increases, one must suspect diastematomyelia. This happened in three children in this series. It was confirmed by myelograms, and the bony spur was removed, with arrest of the progressive paralysis.

\section{Knee in Spina Bifida}

The flexion deformity is important because it may hinder rehabilitation. It is not caused by muscle imbalance but by a faulty posture, spasticity, or fibrosis of the hamstrings. Conservative measures usually suffice, and up to $30^{\circ}$ of flexion deformity can be compatible with ambulation if an appliance is to be worn. In other cases hamstring lengthening and preferably transfer to the patella was found satisfactory.

Genu recurvatum often occurs in lesions at the level of the fourth lumbar segment, when there is hamstring paralysis and a strong quadriceps. It can be prevented by splintage and does not interfere with walking. Elongation of the quadriceps was not found necessary.

\section{Spinal Deformities}

The scar overlying the myelomeningocele is usually quite healthy. There are often associated naevi or lipomata. In most cases the bony gap can be felt and frequently there is local kyphosis. This deformity makes the overlying skin or scar vulnerable to pressure and to avoid this the walkingappliance has to be fitted accordingly. Severe spinal deformities were present in 41 cases: 20 scoliosis, 11 kyphoscoliosis, and 10 kyphosis. Only conservative treatment was required, such as special braces and straps fitted to the walking-appliance. So far no spinal fusion or spinal osteotomies have been performed.

The lumbar lordosis was often combined with a flexion deformity of the hips and was relieved by the flexor-release operation of the hips. Any residual scar or swelling at the site of the myelomeningocele was protected by the lordosis.

\section{Fractures in Spina Bifida}

Fractures often occur in cases of myelomeningocele: they are often undetected, and they heal spontaneously with excess callus formation. They may be an incidental finding when an $x$-ray examination is carried out for some other reason. Prolonged plaster immobilization of paralysed legs causes profound demineralization of the skeleton, and this is often followed by fractures. Soon after removal of the plaster cast the affected limb swells, and this is usually the only clinical sign of a fracture. Both femora and tibiae can thus break after removal of a plaster spica, in spite of careful handling of the limbs. Such complications are rare after the use of splints; the little move- ments these splints permit seem to prevent the severe osteoporosis. Splints are therefore to be preferred to plaster, which should be kept on for the minimum possible time.

Epiphysial separations are less common, but have presented diagnostic difficulties. They may be mistaken for osteomyelitis or even bone sarcoma. The trauma is mild, an ill-fitting calliper may be the cause, bruising is absent, and there is no pain. The radiological signs may be unusual-widening of the epiphysial line, sclerosis, and debris in the soft tissues.

Treatment of fractures presented no problem; they healed readily, requiring protection by splints for only short periods. In this series 20 children had 36 fractures. One epiphysial separation healed slowly and with deformity.

\section{Sores in Spina Bifida}

Sores used to be a dreaded and almost inevitable complication in these cases. If insensitive skin is exposed to prolonged pressure, both from within (bony prominence) and from without (chair, bed, calliper, shoe), ischaemic necrosis will result, giving rise to the sore. Of all the factors operative in its formation, the time factor can be most readily altered. With active rehabilitation and early ambulation the endangered skin is exposed to pressure for short intervals. Sores are therefore not as common or as severe as in the bedridden or chair-fast child. In this series they were found at one time or another in 30 children.

\section{Conclusion}

An attempt has been made to elaborate the problems of the orthopaedic care and rehabilitation of this increasing group of congenitally paraplegic children. The question of schooling and training for employment is becoming acute. In the past the few educable survivors were absorbed into special schools, some entered ordinary schools, and many had no systematic education. At present there is a need for the speedy establishment of special schools for these children. The future may bring further advances in many fields, such as power-driven callipers, improved design of wheel-chairs, and electrical stimulation of the bladder. With all modern methods of treatment, of rehabilitation, and of education, it is hoped that many children will eventually be trained to be of some use in their homes and for some form of suitable employment. This may well bring self-respect and happiness to them and their families.

\section{Summary}

The orthopaedic problems of 173 cases of myelomeningocele are analysed. A plea is made for early ambulation with the help of a walking-appliance which is often successful even in the severest forms of paralysis. Such active treatment helps the physical and mental development of these children and of ten prevents complications such as fractures and sores.

My thanks are due to Mr. J. Atherton, of M. Masters \& Sons Ltd., and Mr. S. Edney, of the Hamlin Surgical Appliances Co., for constructing the appliances and for many helpful suggestions; also to Mr. M. Bayliss for the photographic work.

\section{BIBLIOGRAPHY}

Eckstein, H. B., and Macnab, G. H. (1966). Lancet, 1, 842.

Golding, C. (1960). f. Bone ft. Surg., 42B, 387.

James, C. C. M., and Lassman, L. P. (1962). Ibid., 44B, 828.

Jones, G. B. (1954). Ibid., 36B, 375 .

Mawdsley, T., Rickham, P. P., and Roberts, J. R. (1967). Brit. med. f., $1,663$.

Sharrard, W. J. W. (1964). F. Bone ft. Surg., 46B, 426.

Zachary, R. B., Lorber, J., and Bruce, A. M. (1963). Arch. Dis. Childh., 38, 18.

Smithells, R. W. (1965). In Proceedings of a Symposium on Spina Bifida, p. 2a. London.

Soutter, Felicity E. (1962). F. Bone ft. Surg., 44B, 106. 